\title{
Philosophiques
}

\section{Un nouveau paradigme : le corps sportif}

\section{Léo-Paul Bordeleau}

Volume 12, numéro 1, printemps 1985

URI : https://id.erudit.org/iderudit/203270ar

DOI : https://doi.org/10.7202/203270ar

Aller au sommaire du numéro

Éditeur(s)

Société de philosophie du Québec

ISSN

0316-2923 (imprimé)

1492-1391 (numérique)

Découvrir la revue

Citer cet article

Bordeleau, L.-P. (1985). Un nouveau paradigme : le corps sportif. Philosophiques, 12(1), 33-51. https://doi.org/10.7202/203270ar

\section{Résumé de l'article}

Le corps humain est une réalité plurielle. En tant que tel, il est devenu un pôle de référence pour une nouvelle positivité. De tous les lieux d'expression et de récupération du corps, le sport est devenu le plus accaparant et le plus efficace. Le système sportif façonne le corps humain dans tous ses aspects et lui confère un statut nouveau, celui de corps sportif, ce nouveau paradigme des temps actuels. d'utilisation que vous pouvez consulter en ligne.

https://apropos.erudit.org/fr/usagers/politique-dutilisation/ 


\title{
UN NOUVEAU PARADIGME : LE CORPS SPORTIF
}

\author{
par Léo-Paul Bordeleau
}

\begin{abstract}
RÉSUMÉ. Le corps humain est une réalité plurielle. En tant que tel, il est devenu un pôle de référence pour une nouvelle positivité. De tous les lieux d'expression et de récupération du corps, le sport est devenu le plus accaparant et le plus efficace. Le système sportif façonne le corps humain dans tous ses aspects et lui confere un statut nouveau, celui de corps sportif, ce nouveau paradigme des temps actuels.
\end{abstract}

ABSTRACT. The human body is a multifaceted reality. As such, it has become a reference pole for a new positivity. Among the various areas of corporeal expression and recuperation, sport has become the most absorbing and efficient. Sport as a system shapes the human body in all its aspects, and confers on it a new status : the " corps sportif " which is a new paradigm of our civilization.

\section{A. UN CORPS PLURIEL}

L'actualité du corps n'étonne plus. Une sorte d'inflation tant des praxis du corps que des discours sur le corps envahit la plupart des secteurs de nos sociétés techno-industrielles. Aujourd'hui en effet, le corps ne se contente pas d'exister tout bonnement ; partout il s'affirme, il s'affiche, il revendique, il exige comme jamais il n'a osé le faire. Il est devenu le thème obligé de la culture contemporaine.

D'une part, on se convainc que toute réflexion sur le corps est éthique et métaphysique, dans la mesure où elle exprime une valeur, indique une conduite à suivre et détermine la réalité de la condition humaine. Cette conviction se raffermit dans l'idée que tout discours en général, et toute philosophie en particulier, ne peuvent évacuer une réflexion sur le corps sans se condamner à n'être que spéculation stérile. 
N'est-ce pas ce qui détermine, par exemple, la démarche de la plupart des philosophes contemporains européens lorsqu'ils cherchent à renouveler leurs grilles conceptuelles en vue d'une analyse plus pertinente du statut existentiel de l'homme et de sa relation aux autres ? Chez les phénoménologues notamment ${ }^{1}$, tout s'articule sur la manière corporelle d'être au monde de la conscience de l'individu humain. Le corps est ce qui enracine l'individu dans le monde : " mon corps est le pivot du monde " en ce sens que " $j$ 'ai conscience du monde par le moyen de mon corps $»^{2}$; il est le « centre de référence sensible $»^{3}$. Cependant, dans cette perspective, le corps est toujours le corps propre, le corps vécu, celui qui affecte mon individualité pensante. Il est envisagé par rapport à la conscience : " avoir un corps, c'est être le fondement de son propre néant et ne pas être le fondement de son être ; je suis mon corps dans la mesure où je suis ; je ne le suis pas dans la mesure où je ne suis pas ce que je suis ; c'est par ma néantisation (c'est-à-dire par ma conscience) que je lui échappe $"{ }^{4}$. Ainsi, le corps n'existe que dans l'effort pour le penser : dire que « je ne suis pas devant mon corps, (mais que) je suis dans mon corps, ou plutôt (que) je suis mon corps " ${ }^{5}$ implique que ma conscience est coextensive à mon corps ou que celui-ci est coextensif à celle-là ; le corps est « une coupe instantanée sur le devenir de la conscience ${ }^{6}$.

Dans cette optique, la relation de l'homme à son corps est bien mise en lumière. Mais cette relation est d'abord conçue comme une relation subjective, ou plutôt comme une intersubjectivité corporelle; mon corps ne peut se vivre que dans et par le corps d'autrui ; tous les deux, ils constituent un seul et même tissu que Merleau-Ponty appelle métaphoriquement « la chair "; bref, il y a "intercorporéité " ${ }^{7}$. La dimension sociale de la cor-

1. Nous pensons, entre autres, à Maurice Merleau-Ponty (Phénoménologie de la perception), à Jean-Paul Sartre (L'être et le néant), à Paul Ricour (Le volontaire et l'involontaire), à Michel Henry (Philosophie et phénoménologie du corps), à Claude Bruaire (Philosophie du corps), qui s'inscrivent, avec des nuances bien sûr, dans la tradition spiritualiste occidentale marquée par Platon, Aristote, Descartes, Maine de Biran, Husserl, Heidegger.

2. Maurice Merleau-Ponty, Phénoménologie de la perception, Paris, Gallimard, 1945, p. 97.

3. Jean-Paul Sartre, L'être et le néant, Paris, Gallimard, 1943, p. 37.

4. Ibid., p. 375 .

5: Maurice Merleau-Ponty, Phénoménologie de la perception, Paris, Gallimard, 1945, p. 175.

6. Ibid., p. 93, note 2, Maurice Merleau-Ponty fait ici référence à Matière et mémoire de Bergson.

7. Maurice Merleau-Ponty, Le visible et l'invisible, Paris, Gallimard, 1964, p. 1972-204. 
poréité est ainsi soulignée, mais on l'introduit d'une manière abstraite dans la mesure où ces rapports intersubjectifs de corps restent des rapports interindividuels et atomisés. L'élément du conflit n'existe qu'en tant que lutte des consciences par le truchement des corps : l'expérience de l'aliénation " n'est autre que la saisie métaphysique et horrifiée de mon corps pour l'autre ${ }^{8}$. À la limite, le corps est perçu comme un instrument ; il est " l'instrument que je suis", il est " ma facticité d'être aumilieu-du-monde $"{ }^{9}$, et non pas une force productive insérée dans des rapports de production.

En procédant à une réhabilitation ontologique de la corporéité, la phénoménologie prétendait dépasser le dualisme radical du cartésianisme ; en réalité, ne fait-elle autre chose que déplacer le problème fondamental de l'idéalisme ? La dichotomie classique du corps et de l'âme est-elle vraiment surmontée par le fait de dire que la motricité elle-même (le corps) est une intentionnalité originale et que "la conscience est originairement non pas un je pense que mais un je peux ${ }^{10}$ ?

De son côté, le discours théologique, qui s'est toujours appliqué à éclairer la démarche de l'homme dans son intelligence de la foi, en regard d'une pensée chrétienne héritière de toute une tradition de soupçon face aux revendications corporelles, s'efforce aujourd'hui d'ajuster son éclairage en proposant une réflexion renouvelée sur la vie humaine, corporelle de plus d'une manière, et, plus précisément encore, en approfondissant une théologie de la résurrection ${ }^{11}$.

Par ailleurs, l'explosion du corps est encore plus manifeste et significative dans les sciences humaines contemporaines. Toutes, elles ont à voir avec le projet d'une anthropologie du corps. C'est ainsi que, sur la base du discours des sciences biologiques (anatomie, physiologie, médecine), soit pour le pro-

8. Jean-Paul Sartre, L'être et le néant, Paris, Gallimard, 1943, p. 420.

9. Ibid., p. 409.

10. Maurice Merleau-Ponty, Phénoménologie de la perception, Paris, Gallimard, 1945, p. 160.

11. Par exemple : G. Martelet, Résurrection, eucharistie et genèse de l'bomme, Paris, Desclée, 1972 ; M. Renaud, "Philosophie du corps et résurrection des corps ", in Revue théologique de Louvain, 3 (1972), p. 326-333 ; R. Virgoulay, "Phénoménologie du corps et théologie de la résurrection ", in Revue des sciences religieuses, octobre 1980, p. 323-336 et janvier 1981 , p. 52-75. 
longer ou pour le contester, se sont élaborés des discours nouveaux sur le corps humain : celui de l'ethnologie qui s'occupe de repérer les gestes quotidiens dans diverses sociétés, notamment les gestes du travail, de la nourriture, de l'enfantement et ceux commandés par la ritualité des cultures ${ }^{12}$;, celui de l'esthétique qui fait du corps un objet de représentations artistiques et qui, grâce à une sorte de laïcisation, est parvenu à créer aujourd'hui une érotique formelle ou décorporéisante du corps humain. Mais, plus fondamentalement, on retrouve les interprétations psychobiologiques, psychanalytiques, psychosociologiques et sociologiques, qui, chacune à sa manière, font éclater les explications tenues pour inexpugnables de l'anatomie et de la physiologie, ou tout au moins le modèle qui en découle ${ }^{13}$. Dès lors, elles nous apprennent que le corps n'est pas qu'une configuration anatomo-physique; il est tout aussi essentiellement un corps-relation dans sa structure psycho-biologique, un corps libidinal et fantasmé qui n'est rien en dehors du désir inconscient, un corps regardé et jugé, un corps social et mythique.

D'autre part, au plan de la praxis, le corps est devenu, à bien des égards, l'ultime point d'ancrage des conduites humaines. Ce retour du corps se manifeste non seulement dans les milieux effervescents, mais aussi, au plan du quotidien, dans la façon dont les gens perçoivent, ressentent et parlent leur corps. Ainsi, par exemple, le discours publicitaire, grâce aux raffinements de la technique photographique et aux recherches sur le comportement du consommateur, donne le corps en spectacle, en objet de consommation et comme une marchandise. Il mousse l'avènement d'un corps jouissif contre un corps normatif, en l'inscrivant dans un processus d'égotisation du vécu corporel où l'autre est finalement rapporté à soi et utilisé pour soi. Par ailleurs, cet investissement psychique de son corps est fortement suggéré par une abondante littérature sur les groupes d'expression corporelle et de bio-énergie. L'attention portée ici à son corps est celle du soi corporel comme lieu et medium de découverte d'émotions

12. E.T. Hall, La dimension cachée, trad. fr., Paris, Seuil, 1971 ; M. Douglas, De la souillure. Essai sur la notion de pollution et de tabou, trad. A. Guérin, Paris, Maspero, 1971.

13. Michel Bernard, dans Le corps, Paris, Éditions universitaires, 1972, réédité en 1976, nous livre tout l'ensemble du discours historique sur le corps, depuis la fin du XIX ${ }^{e}$ siècle jusqu'à la sociologie récente. 
et de plaisirs, mais aussi de reconnaissance d'autrui par tous les sens à travers des expériences différenciatrices. En outre, un autre symptôme de style corporéiste a fait son apparition dans certains mouvements religieux : il s'agit de mouvements qui se dénomment eux-mêmes néo-pentecôtistes et charismatiques. Ce qui semble prévaloir dans ces expériences charismatiques et groupales, c'est le mode d'expression non verbal et irruptif dont le véhicule principal est la glossolalie. Enfin, dans un registre expressif et institutionnel en apparence très différent des pratiques groupales corporéistes, on retrouve le sport comme forme d'expression corporelle pour le moins ambivalente.

Dès lors, tant au plan de la théorie que de la pratique, le corps tend à devenir un pôle de référence pour une nouvelle positivité. On est en présence d'une sorte d'hypertrophie corporéiste, alimentée et stimulée par une floraison luxuriante de recherches théoriques et techniques, de témoignages exemplaires, de manifestes politiques, dont le but plus ou moins avoué semble être de justifier et de magnifier la place et le rôle du corps dans la vie individuelle et sociale ; mieux encore, de faire de la corporéité l'essence de l'humanité et, partant, la nouvelle source de tout sens et de toute signification.

C'est le rapport sportif de l'individu humain à son corps qui occupera toute notre étude. De tous les lieux d'expression et de récupération du corps, nous prétendons que le sport, plus précisément le système sportif, tend à devenir le plus accaparant et le plus efficace. En effet, dans nos sociétés techno-industrielles, le phénomène sportif se présente comme un phénomène socioculturel massif, et le système sportif comme la forme dominante de l'éducation du corps, avec ses principes et ses modes d'organisation basés sur la recherche de la performance et du rendement maximal. Par sa prégnance émotionnelle, rationnelle et symbolique, par son poids agonal et persuasif, le sport moderne s'est imposé non seulement à ceux qui le pratiquent, à ceux qui l'organisent ou qui cherchent à le diriger, mais aussi à ceux qui le dénoncent ou le dénigrent. C'est ainsi que cette prégnance massive du sport envahit tous les secteurs de la vie individuelle et sociale. La sportivisation des activités corporelles n'épargne rien ; elle pénètre non seulement l'allure posturale de ceux qui cherchent à reproduire une image de puissance, de performance 
et de rigueur, mais aussi la mode vestimentaire et le langage, les mentalités et les autres institutions. En ce sens, la sportivisation tend à devenir un style de vie, une manière d'être, une éthique.

L'actualité et l'omniprésence du corps et du sport sont donc des faits incontestables. Davantage encore, on ne peut pas ne pas remarquer la contemporanéité de leur efficace manifestation. Or, cette immense percée conjuguée du corps et du sport n'est pas fortuite. Elle doit être interrogée. De la même manière, le fait que, dans un vaste processus de réappropriation sociale et politique, le corps soit particulièrement pris en charge par l'institution sportive, mérite un examen.

C'est donc dans l'optique d'une appropriation sociale du corps, plus précisément de l'utilisation et de la mise en forme d'un corps pluriel selon les exigences normatives de la société dans laquelle nous vivons, que s'articule notre réflexion. Après une brève description de la mise en forme sociale du corps humain, nous chercherons à montrer qu'il est devenu un corps productif dont la dernière forme, paradigmatique à notre avis, est le corps sportif.

\section{1- Le corps humain, une réalité sociale}

Il y a plusieurs parcours expérienciels du corps. Il y a aussi une pluralité d'interprétations du corps qui se situent à la frontière de l'imaginaire et des stéréotypes socio-culturels. Chacune, selon sa visée, construit un corps qu'elle propose, et, à la limite, qu'elle impose comme modèle.

La tradition occidentale nous a habitués à un certain discours sur le corps dont la rationalité s'est construite à partir de l'observation de l'anatomie des cadavres ${ }^{14}$. Ce discours médicophysiologique saisit le corps soit comme l'objet d'une dissection

14. L'avènement d'une représentation anatomique du corps coïncide avec la pratique d'ouverture des cadavres qui commença à se généraliser en Italie, puis dans toute l'Europe, à partir de 1400 ; cette opération contribua à dissiper les inventions fantastiques de l'imagination alchimique et médicale et à détacher le corps des fausses croyances et des superstitions qui prévalaient antérieurement. À l'opposé de cette conception descriptive et statique du corps, la pensée orientale proposa un modèle dynamique et énergétique découlant - en Chine tout au moins - d'une observation du corps chaud et palpitant des suppliciés. 
anatomique, soit comme un système de forces bio-mécaniques ; dans les deux cas, le corps est réduit à une configuration anatomophysiologique neutre. Or, il est remarquable que cette vision du corps humain s'est toujours prévalue de la vérité de son savoir.

Pourtant, ce discours réducteur est de plus en plus remis en question. En effet, la résolution du corps dans son image de cadavre désigne un phénomène à la fois de retrait et de projection. Un phénomène de retrait, puisque, une fois déraciné de ses mythes et vidé de ses mystères, le corps anatomique perd son obscurité et la mort en est ainsi évacuée en même temps que la vie et la sexualité ; dès lors, il ne cache plus rien qui ne puisse être rendu visible par la dissection, l'inventaire des organes et leur nomination. Démystifié et réduit au statut de mosaïque d'organes et de fonctions, ce corps abstrait et transparent se prêtera à la modélisation ; il deviendra le substrat idéologique de toute la connaissance médicale et, comme nous le verrons, de son appropriation socio-sportive. Un phénomène de projection aussi. Car le corps n'est pas que l'objet neutre des anatomistes et des physiologistes : derrière ce corps comme objet anatomique et physiologique, se dissimule le corps imaginaire du désir. Ce sera au tour de la pensée psychanalytique de démystifier l'objectivité de la représentation anatomique du corps. Avec Freud, la représentation psychanalytique découvre le corps comme le lieu imaginaire des désirs ; il est la scène en laquelle viennent se jouer les fantasmes de l'individu. Mosaïque d'organes et de fonctions, le corps est aussi une mosaïque de zones érogènes. Mieux encore, cet investissement par le désir ne s'accomplit pas uniquement dans les zones érogènes privilégiées. C'est du moins ce que nous enseigne Freud dans son analyse du cas d'Élisabeth von $\mathrm{R} \ldots{ }^{15}$ : cet investissement par le désir s'accomplit aussi dans des attitudes et des postures, mettant ainsi en jeu la totalité du corps. C'est qu'en fait, comme l'a affirmé Freud par la suite, le corps doit être " conçu tout entier comme érogène ${ }^{16}$. Le corps anatomique de la jeune fille révèle et masque un corps libidinal et fantasmé, entièrement investi par le désir.

15. Sigmund Freud, Études sur l'bystérie, Paris, P.U.F., 1956.

16. Sigmund Freud, Abrégé de psychanalyse, Paris, P.U.F., 1950, p. 11. 
Ainsi que l'a bien montré Fédida ${ }^{17}$, la démarche anatomomédicale positiviste traditionnelle est en fait ambivalente: réductrice en ce qu'elle se réfere à un corps qui a perdu son sens vital fondamental, elle laisse paraître en même temps un corps qui incarne paradoxalement une dimension pulsionnelle et fantasmatique. C'est que la vision anatomiste du corps procéderait elle-même d'un fantasme originaire : celui du désir le plus archaïque de voir et de connaitre le corps de l'autre, de l'ouvrir, le pénétrer et le découper ${ }^{18}$. Or, selon Fédida, l'ensemble des actes de l'anatomiste (ouvri, disséquer, observer, dessiner, nommer et ordonner les organes) qui, visiblement, cherchent dans le corps une vérité objective, contribuent en même temps, à l'insu de l'auteur, à satisfaire la curiosité primitive d'aller voir ce qu'il y a dans le ventre maternel ${ }^{19}$. Dès lors, le corps érogène relèverait d'une « anatomie fantasmatique ${ }^{20}$, irréductible à celle définie par le biologiste.

Cependant, l'enseignement de la psychanalyse ne se borne pas à nous révéler un corps fantasmatique. Il nous montre en outre que le corps se dévoile réellement à travers les représentations qui traduisent les fantasmes de notre désir inconscient ${ }^{21}$. En somme, le corps est une organisation systématique de lettres qu'il faut déchiffrer dans sa logique et sa singularité : en tant que source et organe de plaisir, le corps est inscription de notre langage et, inversement, notre langage signifie et s'enracine dans l'expérience érogène de notre rorps. Le corps est donc un code

17. Pierre Fédida, "L'anatomie dans la psychanalyse ", in "Lieux du corps ", Nouvelle revue de psychanalyse, Paris, Gallimard, no. 3, 1971, p. 109-126.

18. Ce désir est le plus archaïque puisqu'il porte et vise originellement le corps même des parents ; c'est ce qu'indiquent les observations et l'hypothèse de Mélanie Klein ( $L a$ psychanalyse des enfants, Paris, P.U.F., 1969) pour qui l'enfant tend inconsciemment à détruire le ventre maternel, à le vider pour détruire du même coup le pénis paternel qu'il retient.

19. Pierre Fédida, "L'anatomie dans la psychanalyse ", in "Lieux du corps ", Nouvelle revue de psychanalyse, Paris, Gallimard, no. 3, 1979, p. 125.

20. Pierre Fédida, Corps du vide et espace de séance, Paris, J.P. Delarge éditeur, 1977, p. 27.

21. S. Freud lui-même, dans Métapsychologie, avait déjà souligné qu'une " pulsion ne peut jamais devenir objet de la conscience, seule la représentation qui la figure en est susceptible. Une pulsion ne peut non plus être représentée dans l'inconscient autrement que par la représentation ». Pour sa part, Jacques Lacan reconnaissait que le fantasme, loin d'être réduit à l'imagination, doit être défini « comme une image mise en fonction dans la structure signifiante " du langage (Écrits, Paris, Seuil, 1966, p. 637). Quant à Serge Leclaire, il importe de " prendre le corps à la lettre ", c'est-à-dire d'apprendre " à épeler l'orthographe du nom composé par les zones érogènes qui la constituent " (Psychanalyser. Essai sur l'ordre de l'inconscient et la pratique de la lettre, Paris, Seuil, 1968, p. 76). 
que le langage masque et révèle à la fois. Or ce corps-langage se constitue primitivement, selon J.C. Lavie. ${ }^{22}$, dans la relation du corps de l'enfant avec celui de sa mère dont la présence ne cesse de se faire sentir tout au cours de la vie adulte. Il renvoie ainsi au désir d'autrui. Autrement dit, de par sa nature libidinale de désir de jouissance et, par conséquent, de désir du désir de l'autre, le corps est un constant appel et une constante référence au corps d'autrui. Or, ce qui dans une relation intercorporelle contribue avant tout à valoriser le corps comme objet de désir, c'est le regard. N'est-il pas, en effet, dans le pouvoir de l'espèce humaine de se laisser capter par des images, image de l'autre dans le monde d'abord, puis image de soi-même dans le miroir ? C'est à la vérification d'une telle hypothèse que s'est employée l'analyse lacanienne, dans la ligne de pensée de Bolk ${ }^{23}$. Dès les premières semaines de sa naissance, l'enfant serait saisi par l'image que lui offre le spectacle de la forme totale du corps de l'autre, qui tiendrait lieu de miroir. Il s'agirait d'un stade capital de l'évolution psychologique de l'enfant, le «stade du miroir ", selon l'expression de Lacan. Cette captation visuelle par l'image de son corps et, par là, la recherche narcissique d'une identification avec les autres, se retrouvent chez l'individu adulte.

Le corps naît donc à sa dimension sociale. Or ici, c'est l'approche psychosociologique qui vient compléter le point de vue de la psychanalyse ${ }^{24}$. Cette approche nous apprend que le corps n'acquiert une réalité pleine et entière pour nous que lorsque notre acceptation du regard judicateur d'autrui permet de construire une image de notre corps en accord avec la configuration fantasmatique dessinée par nos désirs. ${ }^{25}$ Loin d'être

22. J.C. Lavie, " Notre corps ou le présent d'une illusion ", in " Lieux du corps ", Nouvelle revue de psychanalyse, Paris, Gallimard, no. 3, 1971, p. 29-36.

23. J. Lacan, Écrits, Paris, Seuil, 1966. F. Gantheret et Lapassade ont donné une traduction de l'ouvrage principal de Bolk, in Revue frangaise de psycbanalyse, mars-avril, 1951.

24. Les observations de M. Masud R. Khan et l'analyse qu'il présente dans "L'œil entend ", in " Lieux du corps ", Nouvelle revue de psychanalyse, Paris, Gallimard, no. 3, 1971, p. 5369, montrent que, par une conjonction du regard et de l'écoute, l'expressivité et la signification du corps regardé s'enrichissent de la significance et de l'expressivité de sa parole, et inversement.

25. Voir Michel Bernard, Le corps, Paris, Éditions universitaires, 1972, p. 110. Ce philosophe français est le premier à nous livrer tout l'ensemble du discours historique sur le corps et son pouvoir expressif, depuis les Romantiques allemands jusqu'à la sémiologie moderne. On peut lire aussi avec grand intérêt son ouvrage sur L'expressivité du corps, Paris, 1976. 
étrangers à notre réalité corporelle, le regard et le jugement d'autrui contribuent nécessairement à la façonner comme nôtre.

Ce travail de façonnement reste cependant ambivalent ${ }^{26}$. Car le regard est à la fois scrutateur ou observateur et expression de l'existence personnelle et affective du sujet ; il est à la fois perception et expression. Mais alors, quand un regard devientil aliénant ou est-il perçu comme tel ? Le regard n'objective et n'aliène le corps d'autrui que lorsqu'il perd toute son expression personnelle et affective : ce regard est purement voyeuriste. $M$. Merleau-Ponty remarque que " nous nous faisons l'un et l'autre regard inhumain, si chacun sent ses actions, non pas reprises et comprises, mais observées comme celles d'un insecte ${ }^{27}$. En réalité, un regard voyeuriste est gênant parce qu'il est unilatéral et bloque une communication possible. Un tel regard pourra même créer des phobies déformatrices, en partie ou en totalité : ce sont les cas d'erythrophobie et de dysmorphophobie ${ }^{28}$.

Ainsi donc, la morphologie corporelle de l'individu, sa configuration fantasmatique, son expressivité aussi, se trouvent modifiées, modelées même, par le regard libidinal et judicatoire de l'autre : non seulement un simple regard attentif, mais un regard qui, pour mieux s'attiser, appelle le vêtement comme écran de l'intimité et comme source de séduction, un regard qui, pour mieux se fixer et ainsi conférer au corps vu une signification déterminée, appelle une raison faiseuse de rituels et de normes sociales. Bref, le regard libidinal et judicatoire, devenu le créneau des exigences normatives d'une société, façonne tout le corps suivant la logique de ces exigences : sa croissance, sa conservation, sa présentation, son expression affective, sa décadence, son inhumation. Il confere ainsi au corps anatomique et fantasmatique une structure sociale et l'élève au rang de mythe

26. J.P. Sartre a formulé à ce sujet une conception sévère des relations intersubjectives, dans L'être et le néant et dans Huis clos, qu'il nuancera dans La critique de la raison dialectique.

27. M. Merleau-Ponty, Phénoménologie de la perception, Paris, Gallimard, 1949, p. 414.

28. Paul Schilder, dans L'image du corps, trad. Gantheret et Truffert, Paris, Gallimard, 1968, a fait une analyse détaillée d'un cas d'erythrophobie comme "névrose sociale ", p. 243250. Des cas de dysmorphophobie sont examinés par S. Tomkiewicz et J. Finded, in "Problèmes de l'image du corps (dysmorphophobie) en foyer de semi-liberté ", Bulletin de psychologie, t. 24, 5-6, 1970-71, p. 263-274. 
culturel $^{29}$. Si l'on reconnaît la validité des analyses de l'anthropologue britannique Mary Douglas, le corps devient un symbole de la société ; il reproduit à une petite échelle les pouvoirs et les dangers qu'on attribue à la structure sociale : " S'il est vrai, écrit-elle, que tout symbolise le corps - c'est l'interprétation de certains psychanalystes, il est tout aussi vrai . . . que le corps symbolise tout ${ }^{30}$.

Cette brève explicitation des parcours expérienciels et discursifs à propos du corps nous interpelle profondément quant à notre manière de parler du corps. Faut-il parler d'un corps ou de plusieurs corps? Il y a effectivement une polysémie du corps. Le corps humain est une réalité qui a l'air très simple, très objective, très physique même, alors qu'en fait on s'aperçoit que des expériences, des disciplines, des sciences extrêmement diverses de par leur point de vue et leur méthode, sont aptes à prendre en charge un certain corps humain. $Y$ aurait-il donc plusieurs corps ? Pourtant, une tradition philosophique occidentale nous a habitués à penser en termes dualistes. Mais cette tradition dans laquelle domine une raison organisatrice et réductionniste, ne reposerait-elle pas sur un malentendu langagier? Rudolf Zur LIPPE nous apprend que « la notion de corps, au commencement de la pensée et de l'histoire européenne, à l'époque de l'antiquité grecque, n'existe pas. Chez Homère, il n'y a pas de notion, il n'y a pas même de mots pour désigner le corps ; en fait, il y en a beaucoup et aucun ${ }^{31}$. À l'époque d'Homère, l'unité du corps (du héros, du guerrier, de l'individu) est constituée par la multiplicité de ses aspects ; l'énergie du corps n'est pas répartie en énergie physique et énergie spirituelle. La dualité n'a pas encore droit de cité. Le dualisme est impensable. Comment sont-ils nés ? Comment la dualité réalité-langage a-t-elle pu engendrer la dualité, puis le dualisme, corps-âme ?

29. Cette structuration sociale du corps a été mise en lumière d'abord par Marcel Mauss, dans "Les techniques du corps ", in Journal de psychologie, t. 32, no. 3-4, mars-avril, 1936. Par la suite, les travaux de recherche de R.M. Birdwhistell et A.S. Hayes, Introduction to Kinesics, University of Louisville Press, 1956, ainsi que les réflexions de E.T. Hall, dans La dimension cacbée, trad. fr., Paris, Seuil, 1971, ont confirmé et nuancé la thèse de M. Mauss.

30. Mary Douglas, De la souillure. Essaie sur la notion de pollution et de tabou, trad. A. Guérin, Paris, Maspéro, 1971, p. 137.

31. Rudolf Zur Lippe, "Une unité problématique : éléments pour une histoire des conceptions du corps ", in Le corps et ses fictions, Paris, Les Éditions de Minuit, 1983, p. 29. 
Ce qu'on nomme « corps » n'est-il pas devenu enveloppe, tombeau, fiction, le moment où l'on a séparé ce qui est physique de ce qui est psychique, c'est-à-dire le moment où le logos en l'homme a décomposé l'unité existentielle de l'être humain ? On sait la facilité qu'a l'individu humain à substantifier son langage. Une sémantique du corps devrait contribuer à clarifier notre problème et à mieux nous interroger sur le statut du corps humain.

Il reste cependant que la pluralité des expériences et des interprétations contemporaines du corps nous invite à relativiser l'idée que nous avons du corps humain. Dès lors, on se saurait confondre le corps ni avec sa réalité anatomo-physiologique, ni avec sa réalité libidinale et fantasmée. Il est aussi une structure sociale $^{32}$. On comprend mieux maintenant pourquoi, dans nos sociétés techno-industrielles de production et de consommation systématiques, le corps est devenu le plus bel objet de consommation $^{33}$. Il l'est devenu parce qu'il est à la fois une structure anatomo-physiologique, une structure libidinale et fantasmée, une structure sociale et mythique. De par sa structure anatomo-physiologique, il est comparé à une machinerie d'organes et de fonctions dont la puissance est harnachée et contrôlée par une raison technicienne ; il est alors conçu comme un mécanisme susceptible d'entretien, appelant une évaluation de ses potentialités et une vérification de son fonctionnement. De par sa structure libidinale et fantasmée, et en tant que mosaïque de zones érogènes, le corps est le lieu du désir et de l'agressivité ; cette mosaïque libidinale profitera cette fois à une raison spéculaire avide d'images publicitaires marquées du sceau de la marchandise. En tant que structure socio-mythique, le corps physique et fantasmatique devient le reflet et le symbole du corps social : agir sur le corps individuel, c'est agir sur l'ensemble social ; le maîtriser, le dompter, le façonner à l'image d'une raison socio-politique, c'est régner sur lui comme sur l'ensemble

32. En réalité, et pour éviter une présentation du corps en pièces détachées, on devrait dire que le corps humain est ni l'une ni l'autre de ces réalités, mais plutôt les trois à la fois. Ce qui fait dire à Michel Bernard, dans Le corps, p. 133, que le corps est " ouverture et carrefour du champ symbolique "; ou encore, qu'il « est l'horizon inaccessible et illusoire des mythes qui en parlent et prétendent en donner la vérité. " (p. 135).

33. J. Baudrillard, La société de consommation, Coll. Idées, Paris, Gallimard, 1970, p. 199238. 
social. Or un corps ainsi conçu et utilisé constitue un capital précieux. N'est-il pas alors une force productive ? C'est ce nouvel aspect que nous allons étudier maintenant.

\section{2- Le corps humain, un corps productif}

Si toute production requiert des moyens, au nombre desquels figurent des instruments, la production par les hommes des conditions même de leur existence semble faire de leur propre corps l'instrument privilégié dont toute technique développée procède. C'est du moins ce qui ressort des considérations marxiennes sur la force de travail définie, à titre de marchandise, comme " l'ensemble des facultés physiques et intellectuelles qui existent dans le corps d'un homme, dans sa personnalité vivante, et qu'il doit mettre en mouvement pour produire des choses utiles ${ }^{34}$. Dès lors, puisque toute production est sociale, selon Marx, et qu'aucune production n'est possible sans un instrument de production $^{35}$, on peut comprendre que le corps apparaisse, selon le mot de Marcel Mauss, comme « le premier et le plus naturel instrument de l'homme ${ }^{36}$. Ainsi, la socialisation du corps ne ferait qu'un avec sa conversion en moyen de production.

Le corps humain deviendrait donc un corps productif. Que veut-on dire par là ? Én quoi cela est-il une identité ? Tout corps biologique n'est-il pas productif dans la mesure où il produit, d'une part, sa propre subsistance selon le concept de l'espèce à laquelle il appartient, et, d'autre part, sa descendance selon ce même concept ?

Pour bien cerner cette dimension d'un corps productif, on ne doit plus sortir de la démarche qui nous a montré que le corps humain est forgé par la communication et l'environnement socio-culturel. Le corps ne vit que par l'échange : il pompe l'air et la nourriture en dehors de lui, dans une indispensable appropriation. Mais son entourage pourvoyeur attend qu'on lui res-

34. K. Marx, Le capital, Li. I, chap. IV, in La Pléiade, Paris, Gallimard, 1965, p. 715.

35. K. Marx, Introduction générale à la critique de l'économie politique, in La Pléiade, Paris, Gallimard, 1965, p. 235 et sq. En réponse aux " robinsonades " de quelques penseurs du XVIII ${ }^{e}$ siècle, Marx affirme que "L'idée d'une production réalisée par un individu isolé, vivant en dehors de la société . . . n'est pas moins absurde que l'idée d'un développement du langage sans qu'il y aic des individus vivant et parlant ensemble. " (p. 236).

36. Marcel Mauss, "Les techniques du corps ", in Jourmal de psychologie, t. 32, 1936, p. 365. 
titue autre chose que les déchets de la consommation : il escompte une certaine productivité, un service-en-retour qui ne sera pas nécessairement quantifiable arithmétiquement ou économiquement. Or, le jeu dialectique entre le corps biologique et le corps social n'a pas toujours privilégié la réciprocité de leurs apports. Marx a bien montré que l'individu morcelé dans son corps et dans son esprit est un phénomène des temps modernes : née du capital, c'est la manufacture qui a introduit la division du travail et partant la parcellisation de l'ouvrier ${ }^{37}$.

En fait, c'est le capitalisme qui accomplit d'incorporer le corps biologique au corps social par la médiation d'un troisième corps, le corps productif. Or ici une distinction s'impose : il n'y a pas de synonymie entre être productif et être producteur. Pour mieux saisir cette différence, il importe de discuter encore le concept de production tel qu'il apparaît dans la pensée marxienne. Nous avons déjà souligné la détermination essentiellement sociale de la production : c'est tout un de dire que l'être de l'homme requiert la production et de dire que l'homme n'a d'être que dans. sa relation aux autres. En d'autres termes, le concept de production circonscrit l'originalité fondamentale de l'existence humaine ; il vient préciser la manière dont l'homme existe au monde. Pour que l'homme soit producteur, ça suppose qu'il puisse prendre connaissance des significations de la réalité et qu'il comprenne la disposition de celle-ci ; ce qui implique que l'homme distingue l'idéalité du sens et la réalité de la matière, et qu'il saisisse, tout au moins intuitionne, la continuité nécessaire de l'idéalité et de la réalité. Dès lors, le concept de production présuppose la praxis, la mienne et celle des autres. Dans la mesure où la praxis définit une structure d'être fondamentale de l'homme, elle est comme l'essence de la production. Toute production est l'actualisation vivante de soi et de l'être-avec-lesautres. La production est une appropriation de soi et du monde. Tel est le sens phénoménologique de la production, suivant lequel tout individu humain est un producteur.

Cependant, dans le système capitaliste, le concept de production acquiert une détermination nouvelle : la production est une production pour l'échange, une production de marchandise,

37. K. Marx, Le capital, Li. I, chap. XIV, in La Pléiade, Paris, Gallimard, 1965. 
c'est-à-dire de produits dont l'exclusive destination est de pouvoir être mis sur le marché. Or, dans cette optique, l'individu qui ne produit pas directement ses moyens de subsistance, mais plutôt des valeurs d'échange, c'est-à-dire des valeurs constituées par un rapport quantitatif et relatives au travail indifférencié et homogène ${ }^{38}$, est un être productif. Être productif n'est pas le propre de qui transforme ou informe une matière préalablement fournie par une activité de travail qui exprime pour elle-même l'individualité du travailleur. Être productif, c'est être susceptible de donner lieu à des produits, objets d'une appropriation. Or, tout produit est fait pour un consommateur éventuel : « la consommation est elle-même un facteur interne de l'activité productive ", elle se présente "comme un moment de production $" 39$.

Tel est le statut du corps humain au sens où nous l'avons présenté, c'est-à-dire une structure concrète, anatomique et physiologique, libidinale et fantasmée, sociale et mythique. En effet, le corps humain devient un corps productif lorsqu'il est, dans toutes ses dimensions, une force pour l'échange, un produit pour être consommable. Or, ce statut de corps productif est imposé au corps humain par les structures mêmes de la production/ consommation que surdéterminent les stratégies du discours publicitaire et de la mode. Ce discours, qu'on peut qualifier de culturaliste parce qu'il est une construction symbolique, véhicule un double objectif pour aboutir à des fins mercantiles de bienheureuse consommation. Il s'agit, d'une part, d'investir psychiquement son corps, en apprenant à l'écouter, à le lire, à se réconcilier avec lui, à l'habiter comme une sorte de résidence secondaire. À cette fin, toute une liturgie de la sollicitude est mise en œuvre, dans le double sens du mot : celui de prendre soin et de gratifier, exprimant ainsi l'idée de gratuité et de don, celui d'exiger et de manipuler pour mieux exploiter. Les canons

38. K. Marx, dans Critique de l'économie politique, in La Pléiade, Paris, Gallimard, 1965, p. 280 : "Les différentes valeurs d'usage sont en outre les produits de l'activité des différents individus, donc le résultat de travaux individuellement différents. En tant que valeurs d'échange, elles représentent cependant du travail homogène, indifférencié, c'està-dire du travail dans lequel l'individualité des travailleurs est effacée. Par conséquent, le travail qui crée la valeur d'échange est du travail général abstrait. "

39. K. Marx, Introduction générale à la critique de l'économie politique, in La Pléiade, Paris, Gallimard, 1965, p. 247. 
de beauté fonctionnelle, une mystique de la ligne, la fascination de la minceur, le culte de la forme et de la performance, l'obsession de virilité/féminité constituent autant d'impératifs capables d'assouvir un regard prédateur et de tisser subtilement dans l'inconscient individuel et collectif une sorte de surmoi marqué du sceau de la marchandise. C'est ainsi que, d'autre part, l'investissement narcissique orchestré se convertit en un investissement économique. Le corps, devenu le plus bel objet de sollicitude et de sollicitation, monopolise à son profit toute l'affectivité et toute l'attention, sans pour autant prendre de valeur propre. Comment le pourrait-il, puisque, dans ce procès de détournement, il n'est pas vécu comme un foyer de désir, mais exploité comme le support objectif de tous les autres objets. Les valeurs d'usage du corps, au service des finalités autonomes du sujet, sont toutes réduites à une valeur d'échange fonctionnelle, selon les objectifs d'une logique productiviste. Le corps érotisé où prédomine la fonction sociale d'échange se substitue au corps libidinal et fantasmé où prévaut la structure individuelle du désir. En somme, il en est du corps comme de la force de travail : il faut qu'il soit émancipé pour pouvoir être rationnellement exploité à des fins mercantiles. "Il faut, précise Jean Baudrillard, que l'individu se prenne lui-même comme objet, comme le plus beau des objets, comme le plus précieux matériel d'échange, pour que puisse s'instituer au niveau du corps déconstruit, de la sexualité déconstruite, un processus économique de rentabilité ${ }^{40}$.

Si l'on s'en tient à ce point de vue, on doit reconnaître que le corps humain n'est pas tant une force productive qu'une puissance ou une faculté de produire, qui n'a sa raison d'être - en partie tout au moins ${ }^{41}$ - que pour et par ses produits, sans en être le propriétaire, puisque être tel suppose le pouvoir de dominer la production, de s'approprier les produits et par là même

40. J. Baudrillard, La société de consommation, Paris, Gallimard coll. Idées, 1970, p. 211. Notre bref exposé sur le corps comme objet de consommation est redevable à l'analyse judicieuse de J. Baudrillard. On peut consulter aussi l'article de Jocelyne Milfort : " Le corps dans la publicité ", in Éros et changement, Paris, Payot, 1981, p. 37-48.

41. Quelle que soit la façon dont on interprète et dont on utilise le corps, celui-ci reste toujours rebelle et irréductible à sa représentation et à son utilisation. Pour le conjurer et le posséder, on l'identifie et on le nomme ; mais ce sont des procédés rationnels fictifs, pourtant utiles pour rendre intelligible un objet - le corps - dont la naturalité n'est qu'un effet de fiction. 
le marché. Dès lors, le corps humain en tant que corps productif n'existe que dans une économie de marché ou un monde de production marchand. Or, dans un tel régime mercantile, la productivité du corps productif s'accroîtra d'autant plus qu'on le fractionnera en forces ou en tâches spécialisées. Or, de même que la manufacture avait introduit la parcellisation de l'ouvrier, en poussant la tendance du métier à se spécialiser, de la même manière elle contribue à l'éclatement du corps afin de le rendre plus productif. La manufacture est par essence hétérogène ; sa raison d'être et son rôle, c'est de séparer la production en ses divers procédés spéciaux dont chacun se cristallise comme tâche particulière. Cependant, cette division manufacturière n'est pas hétéroclite ; elle est systématique en ce sens qu'elle rassemble des tâches ou des métiers différents pour les faire travailler par coopération mécanique à un seul produit ou à un seul objectif.

Tel est le sort du corps humain, devenu corps productif et mercantilisé. Pour répondre aux exigences d'une logique productiviste, il faut que le corps soit déconstruit, réduit à l'état de mosaïque d'organes, de forces, de besoins et de désirs. C'est ainsi qu'on prélève, sélectionne, dresse, chiffre jusqu'à la virtuosité inepte de la tâche parcellaire, ses énergies et ses pouvoirs qui sont susceptibles de devenir des éléments productifs ; et la nouvelle unité qui lui sera attribuée sera toute fonctionnelle, d'essence machinique et mathématique : beauté fonctionnelle, érotisme fonctionnel, performance fonctionnelle, santé fonctionnelle, minceur fonctionnelle, gestualité fonctionnelle d'un corps fonctionnel, c'est-à-dire d'un objet adapté à un ordre ou à un système. La fonctionnalité est ici définie au sens d'une faculté de s'intégrer à un ensemble ${ }^{42}$. Ainsi, pour que se construise le corps productif, il faut que s'effectue un morcellement du corps biologique et fantasmé, que celui-ci devienne une mosaïque de fonctions, non pas en rapport exact au monde réel et aux besoins de l'individu humain, mais en rapport avec un système de représentations interchangeables, que l'unité perdue ne puisse se retrouver que dans une appréhension épistémique qui n'exclut pas la parcellisation des tâches. Dès lors, le corps est soumis au compte des pertes et profits : ce qui est perdu, c'est l'expérience

42. Jean Baudrillard, Le système des objets, Paris, Gallimard, 1970, p. 89. 
et la valeur propres du corps subjectif ; ce qui est promu, c'est l'identité d'un être susceptible de représentations manipulables. Par une ruse souveraine de la raison techno-industrialiste, le corps comme sphère de plaisir autonome est soumis à une sorte de désublimation répressive, nécessaire et indispensable complément d'une nouvelle sublimation qui le hisse au rang de premier instrument cybernétisé ${ }^{43}$.

Ainsi déconstruit, le corps biologique vient à occuper la place d'une mécanique susceptible d'entretien et appelant une évaluation de ses potentialités et une vérification de son fonctionnement. Rompu au service du machinisme industriel, devenu lui-même machinique pour répondre aux objectifs de la recherche exacerbée d'une production toujours plus grande, le corps humain peut désormais être programmé dans toutes ses énergies fragmentées.

On comprend maintenant que le corps humain ne soit plus qu'une réalité subjective, et pas même un objet naturel ; il est aussi et plus évidemment un objet historique et social, le produit du travail et de la culture, du machinisme industriel et de la technique. La signification du corps humain est donc aussi celle de sa place dans le procès de production capitaliste ${ }^{44}$, au moyen d'une formalisation des gestes et d'une rationalisation du mouvement.

Or, si paradoxal que cela puisse paraître, le travail industrialisé n'est pas le seul responsable de cette mécanisation répressive du corps. Dans ce vaste processus de rationalisation, le système sportif contribue tout autant, et plus même, comme nous tenterons de le montrer, à renforcer la construction d'un corps productif par un façonnement qui s'approprie toutes les puissances énergétiques du corps : énergies anatomopsysiologiques, énergies agressives, énergies libidinales et imaginaires. Ce faisant, le système sportif s'impose comme le fil conducteur d'une idéologie de l'efficacité et d'une culturation du corps, contribuant à alimenter l'imaginaire individuel et col-

43. Herbert Marcuse, Éros et civilisation, Paris, Éditions de Minuit, 1963, chap. 10.

44. Il s'agit du procès de production capicaliste d'État que nos trouvons à l'œuvre dans tous les régimes socio-économico-politiques contemporains. 
lectif d'un nouveau modèle chargé d'un pouvoir libidinal, agonistique et formalisant. Ce nouveau corps, nous l'appelons corps sportif.

(à suivre dans notre prochain numéro)

Département de philosophie Université d'Ottawa 